\title{
Gaucher Patient With Bilateral Total Femoral Lytic Lesion Mimicking Malignancy and Review of the Literature Case Report
}

Yavuz ŞAHBAT ( $\nabla$ yavuzsahbat@gmail.com )

Marmara University: Marmara Universitesi https://orcid.org/0000-0002-9963-5334

Ahmet Hamdi AKGÜLLE

Marmara University: Marmara Universitesi

Onur BUĞDAYCI

Marmara University: Marmara Universitesi

Ipek ERBARUT

Marmara University: Marmara Universitesi

Beyza KESKIN

Marmara University: Marmara Universitesi

\section{Research Article}

Keywords: Gaucher disease, Pathological fracture, Diffuse osteopenia

Posted Date: July 29th, 2021

DOI: https://doi.org/10.21203/rs.3.rs-574390/v1

License: (9) This work is licensed under a Creative Commons Attribution 4.0 International License. Read Full License 


\section{Abstract}

Introduction

Bone involvement in Gaucher disease can affect quality of life. Different bone involvements can be confused with hematological diseases, infections and malignancy.

\section{Case}

Our patient with bilateral femur involvement presented to us with a pathological fracture. After the pathological fracture was treated with a long leg splint and healed, we performed biopsy on our patient because of suspicious radiological findings. The pathology results reported gaucher disease with bone infiltration, during the time in which conservative follow up was taking place, eventually leading to the patient's mobilization again.

\section{Conclusion}

The bone findings of gaucher disease portray a difficult process that requires follow-up and treatment. It is crucial to scan patients periodically for possible vertebral and extremity symptoms. Vertebral and extremity fractures are situations that undoubtly require experience as they can imitate malignant masses.

\section{Introduction}

Gaucher Disease (GD) is an autosomal recessive disease due to galactosidase enzyme deficiency; it is categorized as lysosomal storage disorder (LSD) ${ }^{1}$. Gaucher disease affects multiple systems, including the skeletal system. Bone involvement of Gaucher disease may take different forms and affects the quality of life ${ }^{2}$. Skeletal involvement requires treatment for patient mobilization and mortality. In this case report, we present a pathological fracture due to bilateral diffuse femoral osteopenia that may mimic malignancy.

\section{Case}

The patient, who had been followed up for seven years with the diagnosis of Gaucher type 3 by the pediatric genetics and pediatrics gastroenterology clinics, was brought to our emergency department by her family due to bilateral thigh pain during genital cleaning.

The lower extremity motor examination could not be completed due to the patient's diffused anterior thigh pain. It was observed that the lower extremity sensory examination was normal.

Bilateral hands and wrists have a full range of motion. Hand, wrist, flexor and extensor muscle strength were $5 / 5$, and bilateral arms, forearm, and hand sensory examinations were normal. 
X-ray imaging of the patient, who had bilateral proximal thigh pain and tenderness, showed lytic lesions nearly entirely throughout the bilateral femur. In addition, minimally displaced fractures were seen in the right femural diaphysis and in the left femur close to the intertrochanteric area (Fig. 1). X-ray imaging of the bilateral humeral diaphysis showed lytic lesions without pathological fractures. The patient was suffering from chronic low back pain without any significant spinal deformities. Bone pathology and deformity were not seen in the spine radiography (Fig. 2). Bone densitometry performed one year ago was consistent with a 1-4 lumbar Z score of -3.4.

The patient had received imigluserase enzyme therapy two years ago and the pediatric surgery clinic followed up the patient, who had hepatosplenomegaly unresponsive to enzyme replacement therapy.

Patient's laboratory results measured at the time of the fracture; hemoglobin $13.1 \mathrm{~g} / \mathrm{dl}$ (Normal), aspartat aminotransferaz $33 \mathrm{U} / \mathrm{L}(\mathrm{N})$, alanin aminotransferaz $4 \mathrm{U} / \mathrm{L}(\mathrm{N})$, alkaline phosphatase $58 \mathrm{U} / \mathrm{L}(\mathrm{N})$, prothrombin time $19.1 \mathrm{sec}($ High), activated partial thromboplastin time $31.1 \mathrm{sn}(\mathrm{N})$, phosphorus $1.9 \mathrm{mg} /$ dl(Low), magnesium $2 \mathrm{mg} / \mathrm{dl}(\mathrm{N})$, calcium $8.7 \mathrm{mg} / \mathrm{dl}(\mathrm{L}), 25-\mathrm{OH}$ Vit D $7.45 \mathrm{ug} / \mathrm{L}(\mathrm{L})$. In addition, the amount of urinary calcium $109 \mathrm{mg} / \mathrm{dl}$ (High) was determined.

Conservative treatment was decided because the patient's fracture configurations was within acceptable angulation values for 7 years. Bilateral long leg splint with pelvic support was applied with the knees at 15 degrees of flexion.

Callus formations was observed in the second week of control of the patient, whose neurovascular examination was normal, and it was determined to continue splint treatment.

Since mature callus formations was seen at the 4th-week control, splint treatment was discontinued, and passive exercises were started.

At the third month of follow-up, it was found that both knee extensions of the patient were limited by 10 degrees, but the knee flexion muscle strength was $5 / 5$. There was a 100 degrees of flexion and a -10 degrees of extension in both hips of the patient. A total range of motion of 20 degrees was observed in both hips. Pain was reported during FABER and FADIR tests.

Magnetic resonance imaging was recommended due to the diffuse lytic lesions in both femurs on tomography imaging of both femurs (figüre 3 ). A femur biopsy was recommended for the patient with suspected malignancy on contrast-enhanced magnetic resonance imaging. Splenectomy and femur biopsy were performed in the same session with the pediatric surgical team on the patient, who had massive splenomegaly and thrombocytopenia under enzyme replacement therapy for two years.

Phagocytic cells were diffusely observed in the bone marrow biopsy and splenectomy material. These cells, which were found to be PAS positive and immunohistochemically CD68 positive, were evaluated as Gaucher cells with morphological findings (Fig. 4). The patient is still receiving enzyme therapy and vitamin replacement. 
In the 3rd postoperative month, the examination of both hips of the patient was completely pain-free. After 20 sessions of physiotherapy, a significant reduction in spinal pain was achieved. One year after the pathological fracture, the patient came to the control completely without support. X-ray imaging showed complete union accompanied by minimal varus (figüre 5 ).

\section{Discussion}

There are six different bony manifestations of Gaucher disease-bone marrow infiltration, avascular necrosis (AVN), bone crisis, pathological fractures, lytic lesions and osteomyelitis. ${ }^{2}$ Our patient had such a large lytic lesion that pathological fracture was inevitable. In our knowledge, we could not find such a large lesion reported in the literature. In Gaucher's disease, the bones have a less perfect structure than normal bones and are exposed to a higher pathological fracture rate than the normal population. ${ }^{3}$ This means our patient will experience the risk of fractures repeatedly throughout life. It is essential to inform the family about this issue.

The orthopedic problems that develop in types 1 and 3 diseases vary among patients. ${ }^{4}$ Trabecular resorption develops as a result of bone marrow infiltration, cellular necrosis and fibrous proliferation. These findings are reflected on radiographs as focal radiolucent areas in the bone, endosteal combing (cortical thinning) and cortical thinning. In our case, the widespread radiolucent area caused by trabecular resoption was seen in both femurs. Axial skeletal involvement, epiphyseal involvement and Erlenmeyer deformity are common in Gaucher patients. We did not encounter any of them in our patient.

Serum calcium, phosphorus and parathyroid hormone levels are normal in most patients. Urine calcium and hydroxyproline levels are generally normal ${ }^{5}$. The high urinary calcium excretion explains the osteopenia and large lytic lesion in our patient.

X-ray imaging is the first imaging modality to be used, as it provides quick and easy identification of fractures and lytic lesions. After the fracture, the amount of displacement, level of union and follow-up of our patient was done by x-ray. However, when we evaluated our patient in our tumor council, we decided to go for biopsy since we could not distinguish the common lytic lesion on MRI from a lytic lesion formed by malignant tumor tissue.

The incidence of neoplastic disorders such as lymphoproliferative disease appears to be more common in Gaucher disease patients than in the general population. Nevertheless, lesions of benign etiology that mimic these aggressive processes should be considered in the differential diagnosis, when cortical destruction with coexisting soft tissue is the main finding in these patients ${ }^{6}$. This suspicious appearance in Gaucher patients makes orthopedic surgeons doubt the treatment process. Öztürk et al. presented a lesion mimicking a bone tumor in proximal tibia involvement accompanied by Erlenmeyer deformity in the distal femur. ${ }^{7}$ 
Although there are different treatment models in the literature, conservative treatment is the most reliable method in treating pathological fractures in gaucher patients. The existing difficulty in avoiding anesthesia complications, as well as performing surgery on poor quality bone, inclines that it seems safer to immobilize and follow up with a splint and plaster. Pathological femoral fractures of patients can heal with conservative treatment. Varus angulation is an uncommon complication that may develop. ${ }^{8}$ When we first encountered the fracture, we had a few options; follow-up with a splint, follow-up with a pelvipedal cast, closed reduction and percutaneous fixation or open reduction fixation with or without graft. Considering the patient's age and fracture displacement, the decision of conservative follow-up was more reasonable, and saved the patient from a long pelvipedal cast process by applying a splint. If we did surgery, the lytic lesion in the distal area could be the reason for implant failure. Additionally, the patient was not yet evaluated in terms of malignancy. Fixation methods without resection to a malignant bone tissue will make resection very difficult

\section{Conclusion}

The bone findings of gaucher disease portray a difficult process that requires follow-up and treatment. It is crucial to scan patients periodically for possible vertebral and extremity symptoms. Vertebral and extremity fractures are situations that undoubtly require experience as they can imitate malignant masses.

\section{Declarations}

Conflict of Interest: The authors declare that they have no conflicts of interest.

Financial Disclosure: The authors declare that this study has received no financial support.

Consent: Informed consent was obtained from the mother of the patient dou to mild cognitive delay of patient.

\section{Credit authorship contribution statement}

1. Yavuz Şahbat: Conceptualization, Methodology, Visualization, Supervision, Project administration, Writing - original draft, Writing - review \& editing.

2. Ahmet Hamdi Akgülle: Methodology, Formal analysis, Data curation, Writing - review \& editing, Visualization, Supervision.

3. Onur Buğdaycl: Validation, Writing - original draft, Writing - review \& editing.

4. İpek Erbarut Seven: Validation, Writing - original draft, Writing - review \& editing, Visualization, Project administration.

5. Beyza Keskin: Project administration, Writing - original draft, Writing - review \& editing.

\section{References}


1. Brady RO, Kanfer JN, Shapiro D. Metabolism of glucocerebrosides II. Evidence of an enzymatic deficiency in Gaucher's disease. Biochemical and biophysical research communications. 1965;18(2):2215 .

2. Maas M, Poll L, Terk M. Imaging and quantifying skeletal involvement in Gaucher disease. The British journal of radiology. 2002;75(suppl_1):A13-A24.

3. Lebel E, Itzchaki M, Elstein D, Hadas-Halpern I, Abrahamov A, Zimran A. Skeletal manifestations in Gaucher disease: presentation and treatment. The Israel Medical Association journal: IMAJ. 1999;1(4):267-71.

4. Elstein D, Itzchaki M, Mankin HJ. 11 Skeletal involvement in Gaucher's disease. Baillière's clinical haematology. 1997;10(4):793-816.

5. Pastores GM, Patel MJ, Firooznia $\mathrm{H}$. Bone and joint complications related to Gaucher disease. Current rheumatology reports. 2000;2(2):175-80.

6. Hermann G, Shapiro R, Abdelwahab IF, Klein MJ, Pastores G, Grabowski G. Extraosseous extension of Gaucher cell deposits mimicking malignancy. Skeletal radiology. 1994;23(4):253-6.

7. Öztürk H, Ünsal M, Aydingöz Ü, Koçak N, Gürakan F. Pseudotumor formation in tibia in Gaucher's disease. European journal of radiology. 1998;26(3):284-6.

8. Goldman AB, Jacobs B. Femoral neck fractures complicating Gaucher disease in children. Skeletal radiology. 1984;12(3):162-8.

\section{Figures}




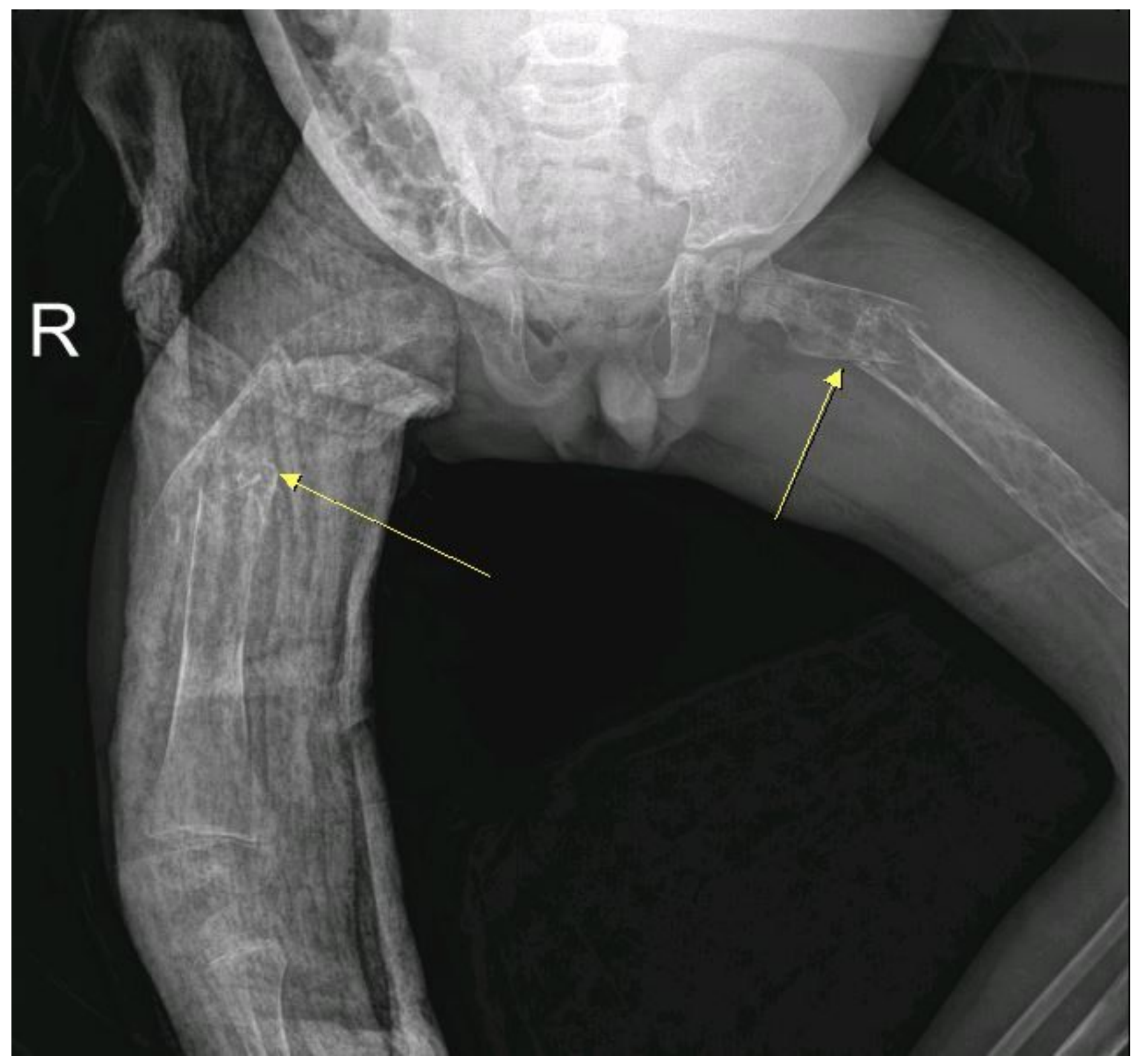

Figure 1

The fracture line in the diaphysis area in the right femur and the fracture line close to the intertrochanteric area in the left femur are shown with an arrow. Diffuse osteopenia is seen in both femurs. 


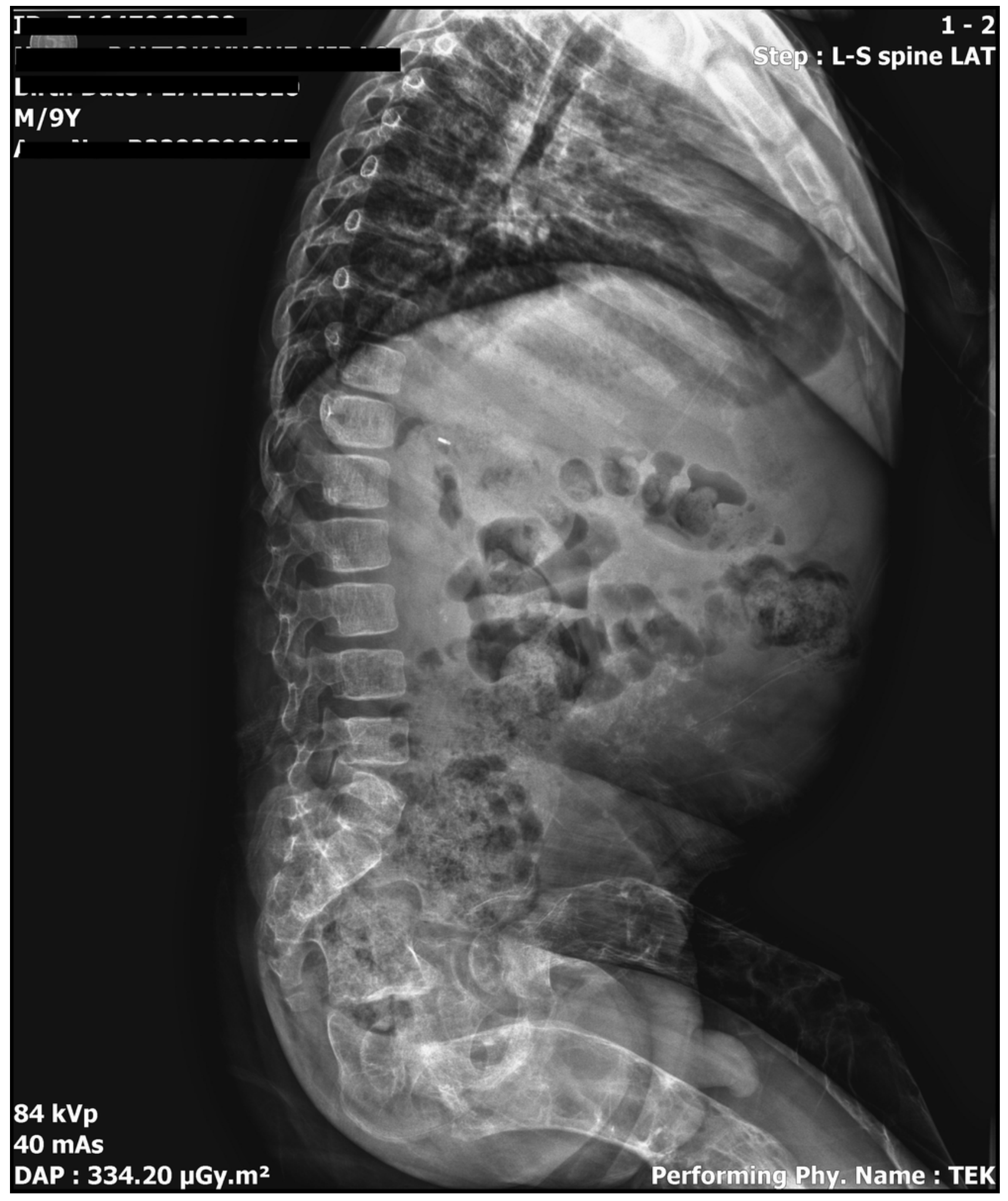

Figure 2

There is no evidence of Gaucher disease related spinal involvement. 


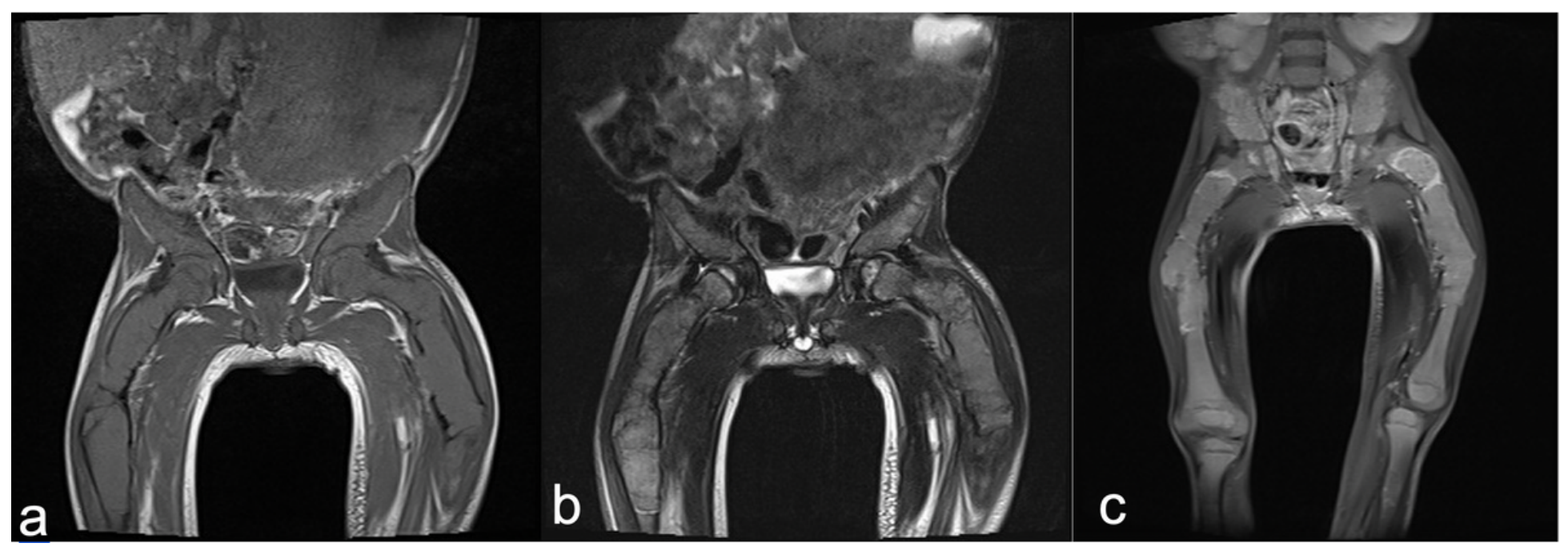

Figure 3

(a) Coronal T1 weighted image shows diffuse involvement of the bone marrow with intermediate signal change. There is diffuse expansion in both femora and pelvic bones. Cortical destruction is present. Deformity is present in both femora (b) Coronal T2 weighted images show heterogenous signal change in the bone marrow. (c) Postcontrast images show slight enhancement at the fracture site in the left proximal femur. There is also slight enhancement in the lef ilium. There is little to no enhancement in other parts, probably due to high cellularity of the bone marrow.

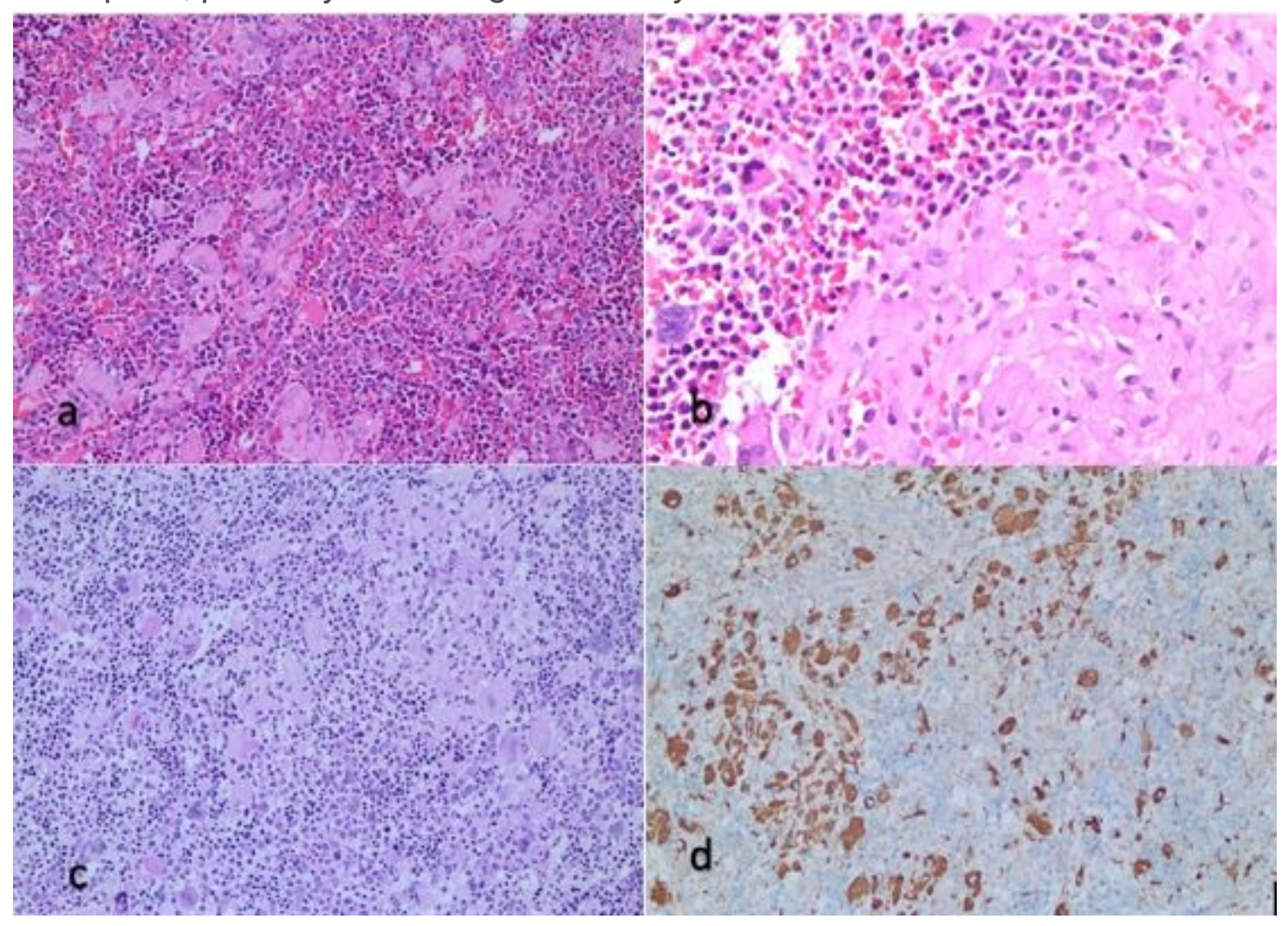

Figure 4 
a. Gaucher cells in bone marrow biopsy are plump macrophages that are characterized by their cytoplasmic appearance of crumpled tissue paper due to glucocerebroside accumulation. b. Gaucher cells under high magnification. c. PAS positivity in Gaucher cells. d. CD68 immunoreactivity in Gaucher cells.



\section{Figure 5}

Bilateral femur remodeling in the 1 st year of fracture development 